\title{
Valor predictivo de la historia clínica y oximetría nocturna en la pesquisa de pacientes con apneas obstructivas del sueño
}

\section{Predictive value of clinical features and nocturnal oximetry for the detection of obstructive sleep apnea syndrome}

Background: Obstructive sleep apnea syndrome (OSA) is an important cause of morbidity and mortality in adults. Aim: To evaluate the diagnostic value of clinical features and oximetric data to screen for obstructive sleep apnea before performing polysomnograpy or respiratory polygraphy. Material and Methods: We studied 328 consecutive adult patients referred for snoring or excessive daytime sleepiness to a sleep clinic in whom a standardized questionnaire and the Sleepiness Epworth Scale were performed and body mass index (BMI), cervical circumference (CC), and nocturnal oximetry were measured. Results: Fifty three percent $(n=173)$ had evidence of clinically significant OSA (apnea/hypopnea index (AHI) $>15$ events/h). Patients with OSA were more likely to be male, obese $\left(B M I \geq 26 \mathrm{~kg} / \mathrm{m}^{2}\right)$, smokers, to have a thick neck $(C C>41 \mathrm{~cm})$, and to have a significant greater prevalence of relative reported apneas and excessive daytime sleepiness, as determined by Epworth scale. Male gender (Odds ratio (OR): 4.00; 95\% confidence intervals (CI): 1.59-10.0, $p=0.003$ ), $B M I \geq 26 \mathrm{~kg} / \mathrm{m}^{2}$ (OR: 3.68; 95\%CI: 1.59-8.49, $p=0.002$ ), smoking (OR: 2.29; 95\% CI: 1.17-4.47, $p=0.015$ ), Epworth index > 13 (OR: 2.65; 95\% CI: $1.35-5.23, p=0.005)$ and duration of symptoms over 2 years (OR: $2.35 ; 95 \%$ CI: $1.20-4.58, p=0.012$ ) were significant independent predictors of OSA. In nocturnal oximetry, the lowest SpO2 (SpO2 min) and the length of registries below 90\% (CT90) were independent predictors of OSA and both correlated significantly with AHI $(r=-0.49$ and $r=0.46$ respectively, $p<0.001)$. Conclusions: No single factor was usefully predictive of obstructive sleep apnea. However, combining clinical features and oximetry data may be appropriate to detect clinically significant OSA patients.

(Rev Med Chile 2010; 138: 941-950).

Key words: Oximetry; Polysomnography; Sleep apnea, obstructive.

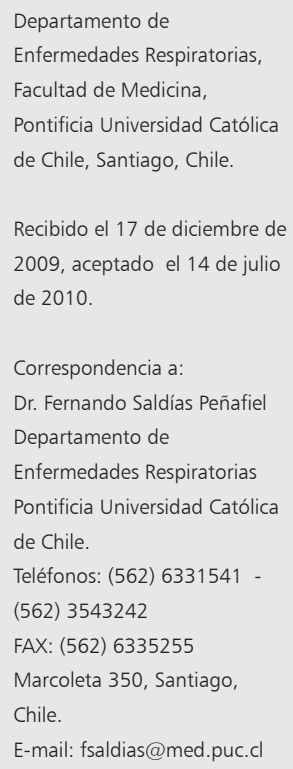

1 1 síndrome de apneas obstructivas del sueño (SAHOS) afecta el 2 a $4 \%$ de la población adulta ${ }^{1}$, ocasionando morbilidad respiratoria, neuropsiquiátrica y cardiovascular significativa ${ }^{2}$. Un tercio de la población adulta ronca ocasionalmente y se estima que 35 a $64 \%$ de los roncadores habituales son portadores de $\mathrm{SAHOS}^{3,4}$. El riesgo de complicaciones se triplica en los roncadores con somnolencia diurna excesiva $^{5}$. La polisomnografía (PSG) es considerada el método de referencia en el diagnóstico de esta condición, pero es un examen laborioso, requiere 
equipamiento complejo, de costo elevado, que no se encuentra disponible en la mayoría de los hospitales $^{6}$. En Estados Unidos, se estima que el 93\% de las mujeres y $82 \%$ de los varones con SAHOS moderado-grave permanecen sin diagnóstico, y las dificultades de acceso a los métodos diagnósticos han impedido resolver este grave problema de salud pública ${ }^{7}$. Por esta razón, se han validado métodos simplificados de diagnóstico que no incluyen las variables neurofisiológicas, entre ellos se encuentra la poligrafía respiratoria (PR) que ha sido ampliamente aceptada como método diagnóstico en grupos seleccionados, disminuyendo los costos y facilitando el acceso al estudio diagnóstico ${ }^{8}$. Además, se ha propuesto la oximetría nocturna como método alternativo simple, económico y accesible, en el apoyo diagnóstico y selección de pacientes con sospecha de apneas obstructivas del sueño, que podría en algunos centros disminuir las largas listas de espera existentes y en otros, poder realizar una adecuada selección de los pacientes para tomar una decisión diagnóstica e incluso terapéutica9.

En la práctica clínica, la decisión de tratar a los pacientes con SAHOS suele estar basada en la magnitud de los síntomas, presencia de comorbilidad cardiovascular y gravedad de los eventos respiratorios (índice de apneas-hipopneas, desaturación y microdespertares nocturnos), ya que estos pacientes se benefician del tratamiento con presión positiva continua en la vía aérea (CPAP) y reducen su riesgo cardiovascular ${ }^{10,11}$. La evaluación sistemática de población oligosintomática y bajo riesgo no se recomendaría, especialmente sujetos sanos sin comorbilidad cardiovascular. Con el propósito de identificar la población de riesgo y focalizar los recursos diagnósticos, se han diseñado y empleado cuestionarios de síntomas que evalúan la magnitud del ronquido, reporte de apneas nocturnas y somnolencia diurna excesiva, hallazgos del examen físico (índice de masa corporal [IMC], perímetro cervical, circunferencia abdominal) y de la oximetría nocturna $\left(\mathrm{SpO}_{2}\right.$ mínima, CT-90, ID$4 \%)$ para la pesquisa de pacientes con SAHOS ${ }^{12-18}$. La sensibilidad y especificidad de los métodos de "screening" para la pesquisa de pacientes con SAHOS ha sido variable, lo cual puede deberse a diferencias de diseño de los estudios, tamaño muestral y evaluación de poblaciones específicas $^{19,20}$. En el medio nacional no existen estudios que hayan evaluado este problema. El propósito de este estudio es evaluar en nuestro medio la utilidad de la evaluación clínica y oximetría digital nocturna en la pesquisa y manejo de pacientes con sospecha de SAHOS.

\section{Pacientes y Método}

Se evaluaron en forma prospectiva los pacientes adultos atendidos en el Centro de Trastornos Respiratorios del Sueño, de la Universidad Católica de Chile, por ronquido intenso y/o somnolencia diurna excesiva entre enero y diciembre de 2004. Antes de realizar el estudio diagnóstico, se aplicó una encuesta estandarizada de síntomas, con el propósito de evaluar el horario de sueño, grado de somnolencia diurna, ronquido, apneas presenciadas, inquietud psicomotora nocturna, insomnio, sofocación nocturna, nicturia, cefalea matinal, boca seca al despertar, pérdida de memoria y deterioro cognitivo. Se consignaron los datos sociodemográficos, comorbilidades, peso, talla y circunferencia cervical medida a nivel de la membrana cricotiroidea. Se evaluó la magnitud de la somnolencia diurna con la escala de Epworth ${ }^{21}$. Se practicó el estudio diagnóstico (polisomnografía o poligrafía respiratoria) y simultáneamente fue realizada una oximetría digital nocturna. El análisis del estudio polisomnográfico y la poligrafía respiratoria fue realizado manualmente por un médico especialista en enfermedades respiratorias o neurología con experiencia en el diagnóstico de trastornos respiratorios del sueño, quienes desconocían los antecedentes clínicos de los enfermos y el resultado de la oximetría nocturna. El estudio fue aprobado por el Comité de Ética de la Institución y los pacientes firmaron el acta de consentimiento informado previo a su incorporación.

\section{Polisomnografía}

El estudio fue realizado a 67 pacientes en el Centro del Sueño con un equipo Alice 3 (Healthdyne Technologies, Ga, EE.UU.), siguiendo las recomendaciones de la Sociedad Americana de Trastornos del Sueño (ASDA) ${ }^{22}$. En todos los pacientes se monitorizaron: a) Variables neurofisiológicas: electroencefalograma con ocho canales, electrooculograma y electromiograma del mentón; b) Variables respiratorias: flujo aéreo oronasal mediante cánula nasal y termistor, movimientos toracoabdominales y saturación de 
Historia clínica y oximetría nocturna en apneas obstructivas del sueño - F. Saldías P. et al

oxígeno $\left(\mathrm{SpO}_{2}\right)$ con un oxímetro de pulso digital. Además, se registró electrocardiograma, posición corporal, magnitud del ronquido y movimientos de las extremidades inferiores mediante electrodos en la cara anterior de las piernas. El registro fue analizado manualmente por un médico entrenado, quien realizó la etapificación de las diferentes etapas del sueño, caracterizó los eventos respiratorios y microdespertares, magnitud del ronquido, cambios posturales y movimientos de extremidades inferiores. Las etapas del sueño fueron analizadas de acuerdo al sistema propuesto por Rechtschaffen y Kales ${ }^{23}$. El análisis del estudio se realizó de acuerdo a las normas de la ASDA empleando las siguientes definiciones ${ }^{22}$ : a) Apnea obstructiva: ausencia del flujo oronasal en presencia de movimientos tóraco-abdominales de más de 10 segundos de duración; b) Hipopneas: reducción del flujo aéreo en más de $30 \%$ por un período superior a 10 segundos asociado a caída de la $\mathrm{SpO}_{2}$ de $4 \%$ o presencia de microdespertares; c) Microdespertar: aparición de onda alfa de 3 y 15 segundos de duración ${ }^{23}$. El índice de apnea hipopnea $(\mathrm{IA} / \mathrm{H})$ se determinó dividiendo el número total de eventos respiratorios por el tiempo total de sueño. El diagnóstico de SAHOS clínicamente significativo fue confirmado por un IA/H mayor de quince eventos por hora de sueño ${ }^{13-17}$ y se definió el SAHOS grave por un IA/H mayor de 30 eventos/ hora de sueño.

\section{Poligrafía respiratoria}

El estudio diagnóstico fue realizado en 261 pacientes con el equipo NightWatch System (Healthdyne, Ga, EE.UU.) que fue validado por Ancoli-Israel y cols, en $1997^{24}$. En el estudio se evaluaron las mismas variables descritas en la PSG con excepción de las variables neurofisiológicas. El análisis del estudio se realizó de acuerdo a las normas de la ASDA empleando las mismas definiciones que en el estudio polisomnográfico. El índice de apnea hipopnea se determino dividiendo el número total de eventos respiratorios por el tiempo total de registro ${ }^{22}$.

\section{Oximetría nocturna}

La oximetría digital nocturna fue realizada con un equipo Ohmeda Biox 3700 (Ohmeda, Louisville $C O, E E . U U$.$) que permite registrar la saturación$ de oxígeno y frecuencia cardíaca cada 12 segundos durante ocho horas, independiente del registro oximétrico asociado a los exámenes antes descritos. El registro fue analizado por uno de los investigadores (FS) en forma independiente al estudio diagnóstico. Se midió la $\mathrm{SpO}_{2}$ basal, $\mathrm{SpO}_{2}$ media, $\mathrm{SpO}_{2}$ mínima, se contabilizó el número de caídas de la saturación mayor de $4 \%$ y se calculó el índice de desaturación de 4\% (ID-4\%), y se cuantificó el porcentaje de la noche que la $\mathrm{SpO}_{2}$ permanecía bajo 80 y $90 \%$ (CT-80 y CT-90).

\section{Análisis estadístico}

Los resultados fueron expresados como valores promedio \pm desviación estándar para las variables medidas en escala numérica y en porcentaje para las medidas en escala nominal. Las variables cualitativas fueron comparadas mediante la prueba de Chi cuadrado y test exacto de Fisher, y las variables continuas con la prueba t de Student. Para ello se utilizaron los programas Epi-Info 6.0 (CDC, Atlanta) y SPSS 12.0 (SPSS Inc., Chicago). Las variables clínicas y oximétricas predictoras de SAHOS fueron sometidas a análisis univariado y multivariado en un modelo de regresión logística (modalidad stepwise) que permite el control simultáneo de múltiples factores. Los parámetros que no agregaron valor predictivo fueron eliminados del modelo. Se calcularon los odds ratio e intervalos de confianza (IC) para el 95\%. Las diferencias entre las variables fueron consideradas significativas con un valor de $\mathrm{p}<0,05$.

Se emplearon las curvas receptor operador (ROC curves) para establecer la sensibilidad y especificidad de las variables clínicas continuas. El análisis de regresión logística nos permitió desarrollar un modelo predictivo de SAHOS basado en las variables clínicas. En el modelo, la presencia o ausencia de la condición es la variable dependiente y la probabilidad de presentar la enfermedad se cuantifica mediante la siguiente fórmula: $p=e^{y} /$ $\left(1+\mathrm{e}^{\mathrm{y}}\right)$, donde: $\mathrm{p}=$ probabilidad de SAHOS, $\mathrm{y}=$ constante $+\mathrm{X}_{1}^{*}$ Variable $_{1}+\mathrm{X}_{2}$ Variable $_{2}+\ldots \mathrm{Xn}$ * Variable $\mathrm{n}$. El análisis de regresión logística fue repetido en los pacientes con SAHOS grave (IA/H mayor de 30 eventos/hora).

\section{Resultados}

Se evaluaron 328 pacientes adultos con historia de ronquido intenso y/o somnolencia diurna excesiva en el Centro del Sueño, la edad promedio 
fue $52 \pm 13$ años (rango: 16-85), 82\% eran varones, $76 \%$ eran obesos (IMC > $25 \mathrm{~kg} / \mathrm{m}^{2}$ ), $28 \%$ fumadores activos y $53 \%$ tenían un IA/H mayor de 15 eventos/h. En las Tablas 1 y 2 se describen las características clínicas y la oximetría nocturna de los pacientes con SAHOS (IA/H: 44,8 $\pm 22,2$ ) y el grupo control (IA/H: $4,7 \pm 4,4)$. Los pacientes con diagnóstico de SAHOS eran de mayor edad $(54 \pm 13$ vs $50 \pm 14$ años, $\mathrm{p}=0,006)$ ), predominio masculino (92 vs 70\%, p < 0,001), tenían mayor
IMC $(\mathrm{p}<0,02)$, perímetro cervical $(\mathrm{p}<0,001)$ y somnolencia diurna excesiva objetivada por la escala de Epworth (13 \pm 6 vs $11 \pm 4$ puntos, $\mathrm{p}<$ $0,001)$. Las comorbilidades fueron similares en ambos grupos.

\section{Características clínicas}

Las variables clínicas asociadas al diagnóstico de SAHOS fueron edad mayor de 45 años (OR: 1,97,p $=0,006)$, sexo masculino (OR: 5,19, $\mathrm{p}<0,001)$, IMC

Tabla 1. Características clínicas de los pacientes adultos atendidos en el Centro del Sueño por ronquido y/o somnolencia diurna excesiva

\begin{tabular}{|c|c|c|c|}
\hline Características & SAHOS & Grupo control & $\mathbf{p}$ \\
\hline $\mathrm{N}$ & 173 & 155 & \\
\hline Edad (años) & $54,3 \pm 12,9$ & $50,2 \pm 13,9$ & 0,006 \\
\hline Sexo $(M-F)$ & $160-13$ & $109-46$ & 0,0001 \\
\hline Mujer postmenopáusica & $10 / 13$ & $22 / 46$ & 0,11 \\
\hline Peso $(\mathrm{kg})$ & $93,1 \pm 20,7$ & $86,0 \pm 20,8$ & 0,004 \\
\hline $\mathrm{IMC}\left(\mathrm{kg} / \mathrm{m}^{2}\right)$ & $31,9 \pm 6,9$ & $30,0 \pm 6,7$ & 0,016 \\
\hline Circunferencia cervical (cm) & $44,0 \pm 3,7$ & $41,3 \pm 4,0$ & 0,0001 \\
\hline Escala de Epworth & $13,1 \pm 5,7$ & $10,7 \pm 4,4$ & 0,0001 \\
\hline Tabaquismo & $114 / 162$ & $79 / 141$ & 0,012 \\
\hline Hipertensión arterial & $73 / 165$ & $60 / 147$ & 0,56 \\
\hline Rinosinusitis crónica & $17 / 164$ & $31 / 143$ & 0,008 \\
\hline Obstrucción nasal crónica & $24 / 164$ & $18 / 143$ & 0,62 \\
\hline Cardiopatía & $35 / 165$ & $38 / 143$ & 0,28 \\
\hline Enfermedad pulmonar crónica & $23 / 165$ & $13 / 143$ & 0,21 \\
\hline Diabetes mellitus & $9 / 165$ & $3 / 143$ & 0,15 \\
\hline Duración de sueño (horas) & $7,2 \pm 1,5$ & $7,3 \pm 1,4$ & 0,57 \\
\hline Ronquido intenso & $158 / 164$ & $137 / 144$ & 0,78 \\
\hline Apneas presenciadas & $136 / 162$ & $94 / 143$ & 0,0003 \\
\hline Somnolencia diurna excesiva & 139/159 & $111 / 140$ & 0,062 \\
\hline Sueño poco reparador & $122 / 157$ & $119 / 139$ & 0,099 \\
\hline Insomnio & $95 / 162$ & $102 / 141$ & 0,016 \\
\hline Sofocación nocturna & $83 / 159$ & $60 / 137$ & 0,16 \\
\hline Inquietud psicomotora nocturna & $102 / 162$ & $89 / 142$ & 1,00 \\
\hline Cefalea matinal & $48 / 161$ & $65 / 142$ & 0,005 \\
\hline Boca seca al despertar & $121 / 159$ & $95 / 137$ & 0,24 \\
\hline Deterioro cognitivo & $104 / 161$ & $104 / 142$ & 0,11 \\
\hline
\end{tabular}

Nota: El número del denominador corresponde a los pacientes con la variable registrada. M: Masculino, F: Femenino, IMC: Índice de masa corporal. 
Tabla 2. Oximetría nocturna de los pacientes adultos atendidos en el Centro del Sueño por ronquido y/o somnolencia diurna excesiva

\begin{tabular}{|lccc|}
\hline Oximetría nocturna & SAHOS & Grupo control & p \\
\hline $\mathrm{SpO}_{2}$ basal (\%) & $94,1 \pm 2,6$ & $94,8 \pm 2,8$ & 0,030 \\
$\mathrm{SpO}_{2}$ media (\%) & $89,6 \pm 6,0$ & $92,7 \pm 4,1$ & 0,0001 \\
$\mathrm{SpO}_{2}$ mínima (\%) & $71,7 \pm 14,9$ & $82,1 \pm 10,6$ & 0,0001 \\
$\mathrm{CT}-90$ (\%) & $35,0 \pm 32,9$ & $15,8 \pm 27,4$ & 0,0001 \\
\hline ID-4\% (eventos/h) & $18,6 \pm 13,1$ & $9,8 \pm 8,8$ & 0,0001 \\
Poligrafía-Polisomnografía & & & 1,00 \\
\hline Duración registro (horas) & $8,1 \pm 0,6$ & $8,1 \pm 0,8$ & 1,00 \\
\hline Eficiencia sueño (\%) & $85,5 \pm 13,2$ & $85,5 \pm 16,0$ & 0,95 \\
\hline Estado vigil (\%) & $14,2 \pm 12,9$ & $14,1 \pm 15,8$ & 0,66 \\
Sueño No REM (\%) & $78,9 \pm 19,2$ & $77,9 \pm 21,5$ & 0,77 \\
\hline Sueño REM (\%) & $10,8 \pm 12,4$ & $10,4 \pm 11,8$ & 0,0001 \\
\hline Índice de apneas-hipopneas & $44,8 \pm 22,2$ & $4,7 \pm 4,3$ & \\
\hline
\end{tabular}

Nota: $\mathrm{SpO}_{2}$ : Saturación de oxígeno, CT-90: tiempo de registro con saturación bajo 90\%, ID-4\%: Índice de desaturación de 4\%.

$\geq 26 \mathrm{~kg} / \mathrm{m}^{2}$ (OR: $\left.3,18, \mathrm{p}<0,001\right)$, circunferencia cervical $>41 \mathrm{~cm}(\mathrm{OR}: 3,72, \mathrm{p}<0,001)$, historia de tabaquismo (OR: 1,86, p < 0,02), duración de los síntomas más de dos años (OR: 2,48, $\mathrm{p}<0,001$ ), apneas presenciadas (OR: 2,73, $\mathrm{p}<0,001$ ), somnolencia diurna excesiva (OR: $1,82, \mathrm{p}=0,062)$ e índice de Epworth mayor de 13 puntos (OR: 2,03, $\mathrm{p}<0,006)$ (Tabla 3).

Las variables antropométricas se correlacionaron con el índice de apneas-hipopneas: peso $(\mathrm{r}=0,24$, IC 95\%: 0,14-0,35, $\mathrm{p}<0,001)$, IMC $(\mathrm{r}=$ 0,18, IC 95\%: 0,07-0,29, p <0,002), circunferencia cervical ( $\mathrm{r}=0,39$, IC 95\%: 0,28-0,48, $\mathrm{p}<0,001)$. El índice de Epworth también se correlacionó con el IA/H ( $r=0,24$, IC 95\%: 0,12-0,34, $p<0,001)$.

\section{Oximetría nocturna}

Los índices obtenidos de la oximetría nocturna asociados al diagnóstico de SAHOS fueron $\mathrm{SpO}_{2}$ basal $\leq 93 \%$ (OR: 1,86, $\mathrm{p}=0,03$ ), $\mathrm{SpO}_{2}$ media $\leq$ 91\% (OR: 3,53, p<0,001), $\mathrm{SpO}_{2}$ mínima $\leq 82 \%$ (OR: 4,76, p < 0,001), CT-90 >15\% (OR: 4,46, $\mathrm{p}<$ 0,001 ), CT- $80 \geq 2 \%$ (OR: $5,87, \mathrm{p}<0,001$ ) y el ID$4 \%>12$ eventos/hora (OR: $3,81, \mathrm{p}<0,001$ ) (Tabla $3)$. En el análisis multivariado fueron predictores independientes de SAHOS la $\mathrm{SpO}_{2}$ mínima $\leq 82 \%$ (OR: 2,62, IC95\%: 1,23-5,54, $\mathrm{p}<0,02)$ y CT-90 $>15 \%$ (OR: 3,92, IC95\%: 1,82-8,40, p < 0,001).
Los siguientes índices oximétricos se correlacionaron con el índice de apneas-hipopneas: $\mathrm{SpO}_{2}$ media ( $\mathrm{r}=-0,45$, IC 95\%: $-0,54 \mathrm{a}-0,35, \mathrm{p}<0,001)$, $\mathrm{SpO}_{2}$ mínima $(\mathrm{r}=-0,49$, IC 95\%: -0,57 a $-0,40, \mathrm{p}<$ 0,001), CT-90 ( $\mathrm{r}=0,46$, IC 95\%: 0,36-0,55, $\mathrm{p}<0,001)$ e ID-4\% ( $r=0,44$, IC 95\%: 0,32-0,54, p < 0,001).

\section{Modelo predictivo}

La sensibilidad y especificidad de las principales variables clínicas y oximétricas asociadas al diagnóstico de SAHOS se exponen en la Tabla 4. En el análisis de regresión logística, las variables clínicas independientes que aportaron al modelo predictivo fueron sexo masculino (OR; 4,00, $p=0,003$ ), IMC $\geq 26 \mathrm{~kg} / \mathrm{m}^{2}$ (OR:3,68, $\mathrm{p}=0,002$ ), índice de Epworth $>13$ puntos (OR: $2,65, \mathrm{p}=0,005)$, historia de tabaquismo (OR: 2,29, $\mathrm{p}=0,015)$ y duración de los síntomas $>2$ años (OR: 2,35, $\mathrm{p}=0,012$ ) (Tabla 5). La probabilidad de un individuo que consulta por ronquido intenso y/o somnolencia diurna excesiva de tener SAHOS (IA/H $>15$ eventos/h) estaría determinada por la siguiente fórmula: $\mathrm{p}=\mathrm{e}^{\mathrm{y}} /\left(1+\mathrm{e}^{\mathrm{y}}\right)$, donde $\mathrm{y}=-3,332+1,389^{\star}$ Sexo $+1,302^{\star} \mathrm{IMC}+$ $0,976^{\star}$ Epworth $+0,83^{\star}$ Tabaquismo $+0,853^{\star} \mathrm{Du}-$ ración de los síntomas. Nótese que la presencia de las variables dicotómicas descritas en la Tabla 5 tiene un valor de 1 y la ausencia un valor de 0 . El resultado del análisis multivariado fue similar en 
Historia clínica y oximetría nocturna en apneas obstructivas del sueño - F. Saldías P. et al

Tabla 3. Índices clínicos y oximétricos predictores de trastornos respiratorios del sueño (SAHOS) en población adulta. Análisis univariado

\begin{tabular}{|c|c|c|c|c|c|}
\hline Características clínicas & Pacientes & SAHOS & OR & IC95\% & $\mathbf{p}$ \\
\hline Edad $>45$ años & 229 & 132 & 1,97 & $1,22-3,19$ & 0,006 \\
\hline Sexo masculino & 269 & 160 & 5,19 & $2,68-10,0$ & 0,001 \\
\hline $\mathrm{IMC} \geq 26 \mathrm{~kg} / \mathrm{m}^{2}$ & 233 & 138 & 3,18 & $1,78-5,68$ & 0,001 \\
\hline $\mathrm{CC}>41 \mathrm{~cm}$ & 190 & 125 & 3,72 & $2,20-6,31$ & 0,001 \\
\hline Duración síntomas $>2$ años & 142 & 91 & 2,48 & $1,46-4,21$ & 0,001 \\
\hline Fumador & 193 & 114 & 1,86 & $1,16-2,99$ & 0,012 \\
\hline Ronquido intenso & 295 & 158 & 1,35 & $0,44-4,10$ & 0,78 \\
\hline Apneas presenciadas & 230 & 136 & 2,73 & $1,58-4,69$ & 0,001 \\
\hline Sofocación nocturna & 143 & 83 & 1,40 & $0,89-2,22$ & 0,16 \\
\hline Somnolencia diurna excesiva & 250 & 139 & 1,82 & $0,97-3,38$ & 0,062 \\
\hline Sueño poco reparador & 241 & 122 & 0,59 & $0,32-1,07$ & 0,099 \\
\hline Insomnio & 197 & 95 & 0,54 & $0,33-0,88$ & 0,016 \\
\hline Cefalea matinal & 113 & 48 & 0,50 & $0,31-0,81$ & 0,005 \\
\hline Deterioro cognitivo & 208 & 104 & 0,67 & $0,41-1,09$ & 0,11 \\
\hline Epworth > 13 puntos & 121 & 78 & 2,03 & $1,25-3,28$ & 0,006 \\
\hline $\mathrm{SpO}_{2}$ basal $\leq 93 \%$ & 74 & 46 & 1,86 & $1,08-3,20$ & 0,030 \\
\hline $\mathrm{SpO}_{2}$ media $\leq 91 \%$ & 103 & 72 & 3,53 & $2,11-5,92$ & 0,001 \\
\hline $\mathrm{SpO}_{2}$ mínima $\leq 82 \%$ & 151 & 103 & 4,76 & $2,88-7,88$ & 0,001 \\
\hline CT-90 > 15\% & 115 & 82 & 4,46 & $2,67-7,47$ & 0,001 \\
\hline $\mathrm{CT}-80 \geq 2 \%$ & 80 & 63 & 5,87 & $3,20-10,7$ & 0,001 \\
\hline ID-4\% > 12 eventos/hora & 95 & 62 & 3,81 & $2,13-6,81$ & 0,001 \\
\hline
\end{tabular}

Nota: OR: Odds ratio, IC95\%: Intervalo de confianza de 95\%, IMC: Índice de masa corporal, CC: Circunferencia cervical, $\mathrm{SpO}_{2}$ : Saturación de oxígeno, CT-80: tiempo de registro con saturación bajo 80\%, CT-90: tiempo de registro con saturación bajo 90\%, ID-4\%: Índice de desaturación de 4\%.

la pesquisa de pacientes con $\mathrm{SAHOS}$ grave $(\mathrm{IA} / \mathrm{H}>$ 30 eventos/hora). En otras palabras, un individuo con las cinco variables clínicas descritas tendría un $88 \%$ de probabilidad de tener SAHOS.

\section{Discusión}

Los principales hallazgos del presente estudio son: a) Las variables clínicas y oximétricas aisladas tuvieron baja sensibilidad y especificidad para establecer el diagnóstico de SAHOS en población de riesgo evaluada en un Centro del Sueño; b) Las variables clínicas combinadas en un modelo matemático permitirían aumentar el valor predictivo de SAHOS, especialmente asociado a los índices oximétricos.

En nuestro estudio, el diagnóstico de SAHOS clínicamente significativo fue definido por un $\mathrm{IA} / \mathrm{H}>15$ eventos/hora ${ }^{12-17}$, ya que en este grupo aumenta el riesgo de complicaciones cardiovasculares y neuropsiquiátricas ${ }^{10,11}$, y se ha demostrado que se beneficiarían del tratamiento con CPAP, lo cual es avalado por las guías internacionales ${ }^{25}$. De hecho, el objetivo de los predictores clínicos es pesquisar a la población de riesgo que se beneficia de tratamiento ${ }^{19,20}$.

Los principales predictores clínicos de SAHOS fueron: edad $>45$ años, sexo masculino, IMC $\geq$ $26 \mathrm{~kg} / \mathrm{m}^{2}$, circunferencia cervical $>41 \mathrm{~cm}$, his- 
Historia clínica y oximetría nocturna en apneas obstructivas del sueño - F. Saldías P. et al

Tabla 4. Sensibilidad y especificidad de las variables clínicas y oximétricas predictoras de trastornos respiratorios del sueño (SAHOS) en población adulta

\begin{tabular}{|c|c|c|c|c|}
\hline Variables clínicas & Sensibilidad & Especificidad & $\begin{array}{c}\text { Área bajo la curva ROC } \\
\qquad \bar{X} \pm E E\end{array}$ & $\mathbf{p}$ \\
\hline Edad $>45$ años & 0,77 & 0,37 & $0,58 \pm 0,03$ & 0,008 \\
\hline $\mathrm{IMC} \geq 26 \mathrm{~kg} / \mathrm{m}^{2}$ & 0,87 & 0,33 & $0,60 \pm 0,03$ & 0,005 \\
\hline $\mathrm{CC}>41 \mathrm{~cm}$ & 0,80 & 0,48 & $0,68 \pm 0,03$ & 0,001 \\
\hline Duración síntomas $>2$ años & 0,69 & 0,54 & $0,60 \pm 0,03$ & 0,009 \\
\hline Epworth $>13$ puntos & 0,50 & 0,67 & $0,62 \pm 0,03$ & 0,001 \\
\hline $\mathrm{SpO}_{2}$ basal $\leq 93 \%$ & 0,32 & 0,80 & $0,58 \pm 0,03$ & 0,016 \\
\hline $\mathrm{SpO}_{2}$ media $\leq 91 \%$ & 0,50 & 0,78 & $0,69 \pm 0,03$ & 0,001 \\
\hline $\mathrm{SpO}_{2}$ mínima $\leq 82 \%$ & 0,72 & 0,65 & $0,74 \pm 0,03$ & 0,001 \\
\hline CT-90 > 15\% & 0,58 & 0,76 & $0,72 \pm 0,03$ & 0,001 \\
\hline CT- $80 \geq 2 \%$ & 0,45 & 0,88 & $0,68 \pm 0,03$ & 0,001 \\
\hline ID-4\% >12 eventos/h & 0,63 & 0,69 & $0,71 \pm 0,03$ & 0,001 \\
\hline
\end{tabular}

Nota: ROC: Curva receptor operador, $\mathrm{X} \pm \mathrm{EE}$ : Promedio \pm Error estándar, IMC: Índice de masa corporal, CC: Circunferencia cervical, $\mathrm{SpO}_{2}$ : Saturación de oxígeno, CT-80: tiempo de registro con saturación bajo 80\%, CT-90: tiempo de registro con saturación bajo 90\%, ID-4\%: Índice de desaturación de 4\%.

Tabla 5. Variables clínicas predictoras de trastornos respiratorios del sueño (SAHOS) en población adulta. Análisis multivariado

\begin{tabular}{|lccccc|}
\hline Características clínicas & Pacientes & SAHOS & OR & IC95\% & P \\
\hline Sexo masculino & 269 & 160 & 4,00 & $1,59-10,0$ & 0,003 \\
IMC $\geq 26$ kg/m² & 233 & 138 & 3,68 & $1,59-8,49$ & 0,002 \\
\hline Duración síntomas $>$ 2 años & 142 & 91 & 2,35 & $1,20-4,58$ & 0,012 \\
Tabaquismo & 193 & 114 & 2,29 & $1,17-4,47$ & 0,015 \\
\hline Epworth $>$ 13 puntos & 121 & 78 & 2,65 & $1,35-5,23$ & 0,005 \\
\hline
\end{tabular}

toria de tabaquismo, duración de los síntomas más de 2 años, reporte de apneas por testigos y somnolencia diurna excesiva objetivada por la escala de Epworth. Estos hallazgos coinciden con los principales predictores clínicos de SAHOS descritos en la literatura, donde destaca la mayor prevalencia en varones y mujer postmenopáusica, obesidad, cuello grueso ( $C C \geq 43 \mathrm{~cm}$ en varones $y \geq 40 \mathrm{~cm}$ en mujeres), historia de ronquido intenso, apneas presenciadas y somnolencia diurna excesiva $^{12-17,19-21}$. En nuestro estudio, las variables antropométricas (IMC, circunferencia cervical) y la escala de Epworth permitieron predecir los eventos obstructivos en pacientes con sospecha de apneas del sueño ${ }^{14,16,17,26,27}$. Además, este trabajo confirma la importancia del tabaquismo como factor de riesgo independiente de SAHOS ${ }^{28}$.

La historia de ronquido intenso y somnolencia diurna excesiva auto-reportada no siempre permite diferenciar a los pacientes con sospecha clínica de SAHOS ${ }^{13,29}$. La somnolencia es confundida por el paciente con fatigabilidad, cansancio y síntomas relacionados a trastornos del ánimo; además, este síntoma es más frecuente en los roncadores habituales sin SAHOS comparado con la población general ${ }^{30}$ y la somnolencia auto-reportada no sería una medición objetiva de esta condición fisiológica $^{31}$. En nuestro estudio no fue factible obtener la información del compañero de dormitorio, ya que el cuestionario fue administrado el mismo día 
que el paciente acudió a realizarse la PSG o PR. Sin embargo, esto no constituyó una gran limitación, considerando que el cuestionario realizado al compañero de pieza sólo aumenta marginalmente el valor predictivo de apneas del sueño ${ }^{15}$.

Se han evaluado varios índices oximétricos en la pesquisa de pacientes con SAHOS, con sensibilidad y especificidad variable que fluctúan entre 40 y $100 \%{ }^{9,17,32}$. En nuestro estudio, el IA/H se correlacionó significativamente con la $\mathrm{SpO}_{2}$ media, $\mathrm{SpO}_{2}$ mínima, CT-90, CT-80 e ID-4\%. Similar a lo descrito por otros autores, los criterios oximétricos fueron mejores predictores de SAHOS que las variables clínicas, con excepción de la circunferencia cervical (ver áreas bajo la curva en Tabla 4) ${ }^{17-20}$.

Se ha cuestionado la utilidad de la oximetría nocturna en la pesquisa de pacientes con SAHOS. Sin embargo, Deegan y cols, evaluaron el valor predictivo del cuadro clínico en 250 pacientes consecutivos referidos a una clínica de sueño y encontraron que los hallazgos clínicos y oximétricos permitieron establecer el diagnóstico en un tercio de los pacientes, los cuales no habrían requerido polisomnografía ${ }^{33}$. La sensibilidad y especificidad de la oximetría nocturna en la pesquisa de pacientes con SAHOS varía ampliamente en la literatura

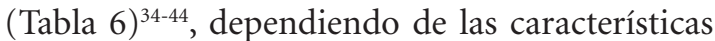
de la población examinada, criterios diagnósticos empleados (índice de desaturación, análisis de la morfología de la curva de $\mathrm{SpO}_{2}$, CT-90, criterios de $\mathrm{SpO}_{2}$ media y mínima) y gravedad de la enfermedad (IA/H > 10 ó 15).

El valor diagnóstico de los criterios clínicos y oximétricos aislados fue sólo moderado y no permitiría utilizarlos como herramienta de tamizaje en población de riesgo. Mientras que la combinación de variables clínicas (edad, sexo, IMC, CC, índice de Epworth) y oximétricas $\left(\mathrm{SpO}_{2}\right.$ mínima, CT-90) y el desarrollo de modelos matemáticos podrían ser de utilidad en la pesquisa de pacientes portadores de SAHOS clínicamente significativo, destinando los recursos diagnósticos y terapéuticos, siempre escasos, a la evaluación y manejo de los pacientes de riesgo elevado ${ }^{12-20}$.

En conclusión, las variables clínicas y oximétricas individuales no permiten establecer con certeza el diagnóstico de SAHOS clínicamente significativo. En cambio, la combinación de variables clínicas y oximétricas podrían ser de utilidad en la pesquisa de población de riesgo y ayudar a orientar los recursos diagnósticos a aquellos pacientes que se beneficiarían de tratamiento.

Tabla 6. Sensibilidad y especificidad de la oximetría nocturna en la pesquisa de pacientes portadores de apneas obstructivas del sueño (SAHOS) ${ }^{34-45}$

\begin{tabular}{|lcccc|}
\hline $\begin{array}{l}\text { Estudio } \\
\text { Autor, año }\end{array}$ & $\begin{array}{c}\text { Población } \\
\mathbf{n}\end{array}$ & $\begin{array}{c}\text { SAHOS } \\
\text { (IA/H) }\end{array}$ & $\begin{array}{c}\text { Sensibilidad } \\
\text { \% }\end{array}$ & $\begin{array}{c}\text { Especificidad } \\
\text { \% }\end{array}$ \\
\hline Cooper et al, 1991 & 41 & $>15$ & 75 & 86 \\
\hline Ryan et al, 1995 & 69 & $\geq 15$ & 31 & 100 \\
\hline Levy et al, 1996 & 301 & $\geq 15$ & 98 & 46 \\
\hline Rodríguez et al, 1996 & 96 & - & 91 & 69 \\
\hline Schafer et al, 1997 & 114 & - & 89,7 & 41 \\
\hline Lacassagne et al, 1997 & 329 & $\geq 15$ & 73,5 & 57,8 \\
\hline Sano et al, 1998 & 40 & $\geq 15$ & 88 & 83,3 \\
\hline Olson et al, 1999 & 113 & $\geq 15$ & 84 & 70 \\
\hline Golpe et al, 1999 & 116 & $\geq 10$ & 85,2 & 97 \\
\hline Nuber et al, 2000 & 70 & $\geq 15$ & 98 & 77,8 \\
\hline Vázquez et al, 2000 & 246 & $\geq 15$ & 94 & 88 \\
\hline Magalang et al, 2003 & 292 & $>15$ & 63 & 44 \\
\hline Saldías et al, 2010 & 328 & & 69 \\
\hline
\end{tabular}

Nota: Los estudios emplearon distintos criterios diagnósticos en la pesquisa de pacientes portadores de SAHOS (índice de desaturación, análisis visual de la curva de $\mathrm{SpO}_{2}$, variabilidad de la curva de $\mathrm{SpO}_{2}, \mathrm{CT}-90, \mathrm{SpO}_{2}$ media y mínima). 


\section{Referencias}

1. Young T, Palta M, Dempsey J, Skatrud J, Weber S, Badr $\mathrm{S}$. The occurrence of sleep-disordered breathing among middle-aged adults. N Engl J Med 1993; 328: 1230-5.

2. Panossian LA, Avidan AY. Review of sleep disorders. Med Clin North Am 2009; 93: 407-25.

3. Gislason T, Aberg H, Taube A. Snoring and systemic hypertension -an epidemiological study. Acta Med Scand 1987; 222: 415-21.

4. Guilleminault C, Tilkian A, Dement WC. The sleep apnea syndromes. Annu Rev Med 1976; 27: 465-84.

5. Lindberg E, Janson C, Gislason T, Svärdsudd K, Hetta J, Boman G. Snoring and hypertension: a 10 year followup. Eur Respir J 1998; 11: 884-9.

6. Flemons WW, Douglas NJ, Kuna ST, Rodenstein DO, Wheatley J. Access to diagnosis and treatment of patients with suspected sleep apnea. Am J Respir Crit Care Med 2004; 169: 668-72.

7. Young T, Evans L, Finn L, Palta M. Estimation of the clinically diagnosed proportion of sleep apnea syndrome in middle-aged men and women. Sleep 1997; 20: 705-6.

8. Thurnheer R, Bloch KE, Laube I, Gugger M, Heitz M. Swiss Respiratory Polygraphy Registry. Respiratory polygraphy in sleep apnoea diagnosis. Report of the Swiss respiratory polygraphy registry and systematic review of the literature. Swiss Med Wkly 2007; 137: 97-102.

9. Netzer N, Eliasson AH, Netzer C, Kristo DA. Overnight pulse oximetry for sleep-disordered breathing in adults: a review. Chest 2001; 120: 625-33.

10. Marin JM, Carrizo SJ, Vicente E, Agusti AG. Long-term cardiovascular outcomes in men with obstructive sleep apnoea-hypopnoea with or without treatment with continuous positive airway pressure: an observational Study. Lancet 2005; 365: 1046-53.

11. Somers VK, White DP, Amin R, Abraham WT, Costa F, Culebras A, et al. Sleep apnea and cardiovascular disease: an American Heart Association/American College of Cardiology Foundation Scientific Statement from the American Heart Association Council for High Blood Pressure Research Professional Education Committee, Council on Clinical Cardiology, Stroke Council, and Council on Cardiovascular Nursing. In collaboration with the National Heart, Lung, and Blood Institute National Center on Sleep Disorders Research (National Institutes of Health). Circulation 2008; 118: 1080-111.

12. Stradling JR, Crosby JH. Predictors and prevalence of obstructive sleep apnoea and snoring in 1,001 middle aged men. Thorax 1991; 46: 85-90.

13. Viner S, Szalai JP, Hoffstein V. Are history and physical examination a good screening test for sleep apnea? Ann
Intern Med 1991; 115: 356-9.

14. Davies RJ, Ali NJ, Stradling JR. Neck circumference and other clinical features in the diagnosis of the obstructive sleep apnoea syndrome. Thorax 1992; 47: 101-5.

15. Kump K, Whalen C, Tishler PV, Browner I, Ferrette V, Strohl KP, et al. Assessment of the validity and utility of a sleep-symptom questionnaire. Am J Respir Crit Care Med 1994; 150: 735-41.

16. Flemons WW, Whitelaw WW, Brant R, Remmers JE. Likelihood ratios for a sleep apnea clinical prediction rule. Am J Respir Crit Care Med 1994; 150: 1279-85.

17. Herer B, Roche N, Carton M, Roig C, Poujol V, Huchon G. Value of clinical, functional, and oximetric data for the prediction of obstructive sleep apnea in obese patients. Chest 1999; 116: 1537-44.

18. Wiltshire N, Kendrick AH, Catterall JR. Home oximetry studies for diagnosis of sleep apnea/hypopnea syndrome: limitation of memory storage capabilities. Chest 2001; 120: 384-9.

19. Pang KP, Terris DJ. Screening for obstructive sleep apnea: an evidence-based analysis. Am J Otolaryngol 2006; 27: 112-8.

20. Ramachandran SK, Josephs LA. A meta-analysis of clinical screening tests for obstructive sleep apnea. Anesthesiology 2009; 110: 928-39.

21. Osman EZ, Osborne J, Hill PD, Lee BW. The Epworth Sleepiness Scale: Can it be used for sleep apnoea screening among snorers? Clin Otolaryngol Allied Sci 1999; 24: $239-41$.

22. Iber C, Ancoli-Israel S, Chesson A, Quan SF, for the American Academy of Sleep Medicine. Westchester, Ill: American Academy of Sleep Medicine; 2007. The AASM Manual for the Scoring of Sleep and Associated Events: Rules, Terminology and Technical Specifications.

23. Rechtschaffen A, Kales A. A manual of standardized terminology, techniques and scoring system for sleep stages in human subjects. Washington, DC, National Institutes of Health, 1968; Publication No. 204.

24. Ancoli-Israel S, Mason W, Coy TV, Stepnowsky C, Clausen Jl, Dimsdale J. Evaluation of sleep disordered breathing with unattended recording: the Nightwatch System. J Med Eng Technol 1997; 21: 10-4.

25. Grupo Español de Sueño. Consenso Nacional sobre el síndrome de apneas-hipopneas del sueño. Arch Bronconeumol 2005; 41 (Supl 4): 3-110.

26. Katz I, Stradling J, Slutsky AS, Zamel N, Hoffstein V. Do patients with obstructive sleep apnea have thick necks? Am Rev Respir Dis 1990; 141: 1228-31.

27. Hoffstein V, Mateika S. Differences in abdominal and neck circumferences in patients with and without obstructive sleep apnoea. Eur Respir J 1992; 5: 377-81. 
Historia clínica y oximetría nocturna en apneas obstructivas del sueño - F. Saldías P. et al

28. Kashyap R, Hock LM, Bowman TJ. Higher prevalence of smoking in patients diagnosed as having obstructive sleep apnea. Sleep Breath 2001; 5: 167-72.

29. Dealberto MJ, Ferber C, Garma L, Lemoine P, Alpérovitch $\mathrm{A}$. Factors related to sleep apnea syndrome in sleep clinic patients. Chest 1994; 105: 1753-8.

30. Bliwise DL, Nekich JC, Dement WC. Relative validity of self-reported snoring as a symptom of sleep apnea in a sleep clinic population. Chest 1991; 99: 600-8.

31. Thorpy MJ. The clinical use of the Multiple Sleep Latency Test. The Standards of Practice Committee of the American Sleep Disorders Association. Sleep 1992; 15: 268-76.

32. Ferber R, Millman R, Coppola M, Fleetham J, Murray $\mathrm{CF}$, Iber $\mathrm{C}$, et al. Portable recording in the assessment of obstructive sleep apnea. ASDA standards of practice. Sleep 1994; 17: 378-92.

33. Deegan PC, Mcnicholas WT. Predictive value of clinical features for the obstructive sleep apnoea syndrome. Eur Respir J 1996; 9: 117-24.

34. Cooper BG, Veale D, Griffiths CJ, Gibson GJ. Value of nocturnal oxygen saturation as a screening test for sleep apnoea. Thorax 1991; 46: 586-8.

35. Ryan PJ, Hilton MF, Boldy DA, Evans A, Bradbury S, Sapiano $S$, et al. Validation of British Thoracic Society guidelines for the diagnosis of the sleep apnoea-hypopnoea syndrome: can polysomnography be avoided? Thorax 1995; 50: 972-5.

36. Levy P, Pepin JL, Deschaux-Blanc C, Paramelle B, Brambilla C. Accuracy of oximetry for detection of respiratory disturbances in sleep apnea syndrome. Chest 1996; 109: 395-9.

37. Rodríguez González Moro JM, De Lucas Ramos P, Sánchez Juanes MJ, Izquierdo Alonso JL, Peraita Adrados R, Cubillo Marcos JM. Usefulness of the visual analysis of night oximetry as a screening method in patients with suspected clinical obstructive sleep apnea syndrome. Arch Bronconeumol 1996; 32: 437-41.

38. Schafer H, Ewig S, Hasper E, Luderitz B. Predictive diagnostic value of clinical assessment and nonlaboratory monitoring system recordings in patients with symptoms suggestive of obstructive sleep apnea syndrome. Respiration 1997; 64: 194-9.

39. Lacassagne L, Didier A, Murris-Espin M, Charlet JP, Chollet P, Leophonte-Domairon ML, et al. Role of nocturnal oximetry in screening for sleep apnea syndrome in pulmonary medicine. Study of 329 patients. Rev Mal Respir 1997; 14: 201-7.

40. Sano K, Nakano H, Ohnishi Y, Ishii Y, Nakamura T, Matuzawa K, et al. Screening of sleep apnea-hypopnea syndrome by home pulse oximetry. Nihon Kokyuki Gakkai Zasshi 1998; 36: 948-52.

41. Olson LG, Ambrogetti A, Gyulay SG. Prediction of sleepdisordered breathing by unattended overnight oximetry. J Sleep Res 1999; 8: 51-5.

42. Golpe R, Jiménez A, Carpizo R, Cifrian JM. Utility of home oximetry as a screening test for patients with moderate to severe symptoms of obstructive sleep apnea. Sleep 1999; 22: 932-7.

43. Nuber R, Vavrina J, Karrer W. Predictive value of nocturnal pulse oximetry in sleep apnea screening. Schweiz Med Wochenschr 2000; 116 (Suppl): 120S-122S.

44. Vázquez JC, Tsai WH, Flemons WW, Masuda A, Brant R, Hajduk E, et al. Automated analysis of digital oximetry in the diagnosis of obstructive sleep apnoea. Thorax 2000; 55: 302-7.

45. Magalang UJ, Dmochowski J, Veeramachaneni S, Draw A, Mador MJ, El-Solh A, et al. Prediction of the apneahypopnea index from overnight pulse oximetry. Chest 2003; 124: 1694-701. 